\title{
The Use of Chiropractic Massage Techniques in Health Care for Children
}

\author{
Liangbing Yang, Ying Shao, Haikuan Wang \\ Guangzhou University of Chinese Medicine, Guangzhou Guangdong, 510006, China
}

Keywords: Chiropractic massage, Child health care, Application effect.

\begin{abstract}
Purpose Analyze the effect of the use of chiropractic massage technique in health care for children. Method Take 90 cases of neonates born in our hospital as the research object, which are divided into A and B groups with 45 cases in each group. Touching group B, and carrying on chiropractic massage on the basis of Group $B$, comparative comparison and analysis of the application effect of the standard massage and chiropractic massage on neonates in the two groups before and after the implementation. Result Before the implementation, comparing the weight, height, sleep time, the total incidence of physical discomfort symptoms and adverse reactions between the two groups, the difference is not significant $(\mathrm{P}>0.05)$. After the implementation, the clinical indicators of Group A, like weight, height, sleep time and so on, is better than Group B; the gain of weight, height, sleep time is greater than Group B; the total incidence of physical discomfort symptoms (4.44\%) is less than Group B (42.22\%); the total incidence of adverse reactions (31.11\%) is less than Group B $(57.78 \%)$, all has distinct difference $(\mathrm{P}<0.05)$. Conclusion Carrying chiropractic and massage technique on neonates in child care, can effectively reduce incidence of physical discomfort symptoms and adverse reactions of the neonatal body, and promote the healthy growth of newborns, which is worth popularizing and applying.
\end{abstract}

\section{Introduction}

Massage is a treatment and health care method for traditional diseases in Chinese medicine theory, which mainly operates on body acupuncture points through a variety of techniques to play a role of compromise on the viscera, fuzheng quxie, dredging shuluo, so as to achieve the purposes of prevention,treatment of diseases and health care ${ }^{[1]}$. And the introduction of chiropractic massage technique in child care, that is, under the condition of no obvious symptoms in infants and young children, carry out its meridian conditioning according to the physiological state, in order to achieve the supplementation of infant body, the release of excess materials, and the adjustment of virtual multi-ferrite ${ }^{[2]}$. In infant health care, chiropractic massage technique can effectively promote the growth and development, and ensure health care. In order to further explore the application of the technology, 45 cases of infants and young children are practiced the technology, and the relevant research is carried out. The report is as follows.

\section{Materials and Methods}

\section{General Information}

From March 2015 to March 2016, we selected 90 cases of newborns received in the outpatients of our hospital as the research object, whose families were informed of it and they all agreed with the operation. The newborns with no dysfunction in major organ, being excluded other complications, diseases or symptoms, who recently had not received the treatment and care, were divided into two groups: group A and group B with 45 cases in each one. Among them, 26 cases of male baby, 19 cases of girl baby in group A; the age of $3 \sim 6$ months, the average of $(30.12+0.87)$ months. 27 cases of boys, 18 cases of girls in group B; the age of $4 \sim 6$ months, the average of $(3.09+0.68)$ months. There is little difference in the comparison of neonatal data in two groups $(\mathrm{P}>0.05)$, which exists comparability. 


\section{Method}

Group B: Carry out standard massage to the neonatal group, specific methods of operation: before touching, the operation personnel should warm hands, and use a amount of baby moisturizing cream evenly coated in the hands of palm, in order to reduce the delicate skin of newborn caused by irritation and friction, reduce the neonatal discomfort; then touch on neonatal back, limbs, abdomen, chest and head and face and other parts, and the massage strength should be moderate, touch action should be as gently as possible, each repeat 3 to 5 times, ongoing 3 months.

Group A:On the basis of Group B,Carry out chiropractic massage on the neonatal group, specific operation method: use thumb knead and chiropractic on neonatal back foot Taiyang bladder meridians and Du meridian points $3 \sim 5$ times for massage and chiropractic chiropractic, point massage $15 \sim 20$ times for each point. Dialectical operation methods: for newborns with the eccentric liver, purges heart meridian and liver for 200 times, spleens liver, lung, kidney 100 times for each one, clears river and intestine 200 times; for newborns with deficiency of spleen and lung, more $3 \sim 5$ times of chiropractic and Cadogan, 150 times of pressing and kneading Banmen, 100 times of pushing off, 300 times of invigorating the spleen, lung, kidney; for newborns with the need for a balance state, 100 times of liver meridian and heart meridian, 200 times of reinforcing and spleen. One time for every 1 days, $5 \sim 10$ min each time, 3 months ongoing.

\section{Outcome Measures}

Before and After the implementation of massage and chiropractic massage, the observe and record the clinical indicators like the sleep time, growth velocity (weight and height), nutritional status of the newborns in two groups, physical discomfort and adverse reaction incidence, and then do the correlation analysis.

\section{Statistical Methods}

Analyze the data by means of statistical software SPSS 21.0, use () to instead of the data in the measurement data, use t test; use (\%) to instead of the data count in data, use the $\mathrm{x} 2$ test, when the comparative data exists statistical significance, indicate with $(\mathrm{P}<0.05)$.

\section{Result}

The comparison of the changes of the two groups' clinical indicators before and after the implementation

Before the implementation, there is no significant difference in the index comparison of two groups like weight, height, and sleep time $(\mathrm{P}>0.05)$; after the implementation, weights of group $\mathrm{A}$ is heavier than those of group B before the implementation, height is higher than those of group B before the implementation, sleep time is longer than those of group B before the implementation, all has a significant difference $(\mathrm{P}<0.05)$. See Table 1 .

Table 1. The comparison of the changes of the two groups' clinical indicators before and after the implementation( $(\bar{X} \pm s$ )

\begin{tabular}{|c|c|c|c|c|}
\hline Groups & Time & Weight(kg) & Height(cm) & Sleep Time(h) \\
\hline \multirow[b]{2}{*}{ Group $A(n=45)$} & Before implementation & $4.87 \pm 0.98$ & $51.21 \pm 2.31$ & $15.09 \pm 0.73$ \\
\hline & After implementation & $\begin{array}{r}6.44 \pm \\
1.21 \mathrm{a} \\
\end{array}$ & $\begin{array}{c}61.68 \pm \\
5.35 \mathrm{a}\end{array}$ & $19.24 \pm 2.10 \mathrm{a}$ \\
\hline \multirow{2}{*}{ Group $B(n=45)$} & Before implementation & $4.89 \pm 0.95$ & $50.86 \pm 2.52$ & $14.97 \pm 0.81$ \\
\hline & After implementation & $5.10 \pm 1.05$ & $55.68 \pm 4.41$ & $17.12 \pm 1.83$ \\
\hline
\end{tabular}

Note: Comparing with Group B before the implementation, $\mathrm{aP}<0.05$.

The comparison of the gain of newborns before and after the clinic indexes

After the implementation, the weight, height and sleep time of group A were higher than that of group $\mathrm{B}$, and there was great difference $(\mathrm{P}<0.05)$. See Table 2. 
Table 2. The comparison of the gain of newborns before and after the clinic indexes( $\bar{X} \pm S$ )

\begin{tabular}{cccc}
\hline Groups & \multicolumn{3}{c}{ Weight Gain $(\mathrm{cm})$ Height Gain $(\mathrm{kg})$ Sleep Time Gain(h) } \\
\hline Group A(n=45) & $2.43 \pm 1.03 \mathrm{a}$ & $10.47 \pm 3.21 \mathrm{a}$ & $4.15 \pm 1.23 \mathrm{a}$ \\
\hline Group B(n=45) & $1.04 \pm 0.34$ & $5.22 \pm 2.11$ & $2.15 \pm 0.89$ \\
\hline \multicolumn{5}{c}{ Note: Comparing with Group B, aP<0.05. }
\end{tabular}

\section{The comparison of the occurrence of physical discomfort symptoms of the newborns in two groups}

After observation, after 3 months, the neonates in the two groups all showed symptoms of anemia, malnutrition, rickets, in which the total incidence rate of discomfort symptoms of newborn in group A was lower than that in group B with significant difference $(\mathrm{P}<0.05)$, as shown in Table 3.

Table 3. The comparison of the occurrence of physical discomfort symptoms of the newborns in two groups(n/\%)

\begin{tabular}{ccccc}
\hline \multirow{2}{*}{ Groups } & Anemia & Malnutritio & \multicolumn{2}{c}{ Rickets Total incidence rate } \\
\hline Group A(n=45) & $1(2.22)$ & $1(2.22)$ & $0(0.00)$ & $2(4.44)$ \\
\hline Group B(n=45) & $\begin{array}{c}7(15.56 \\
)\end{array}$ & $9(20.00)$ & $3(6.67)$ & $19(42.22)$ \\
\hline X2 & - & - & - & 17.95 \\
\hline P & - & - & - & $<0.05$ \\
\hline
\end{tabular}

\section{The comparison of incidence of adverse reactions in the two groups were compared}

During the experiment, the newborns in two groups both occurred adverse reaction of diarrhea, spits, pneumonia and red buttock and so on, the total incidence rate of adverse reactions in group A was lower than that in group $\mathrm{B}$, with significant difference $(\mathrm{P}<0.05)$, as shown in table 44 .

Table 4 . The comparison of incidence of adverse reactions in the two groups were compared(n/\%)

\begin{tabular}{cccccc}
\hline \multirow{2}{*}{ Groups } & \multicolumn{2}{c}{ Diarrhe } & \multirow{3}{*}{ Vomiting Milk } & Pneumoni & \multirow{2}{*}{ Red Buttock Total incidence rate } \\
& a & & a & \\
\hline Group A(n=45) & $2(4.44)$ & $11(24.44)$ & $0(0.00)$ & $1(2.22)$ & $14(31.11)$ \\
\hline Group B(n=45) & $4(8.88)$ & $15(33.33)$ & $2(4.44)$ & $5(11.11)$ & $26(57.78)$ \\
\hline $\mathrm{X} 2$ & - & - & - & - & 6.48 \\
\hline $\mathrm{P}$ & - & - & - & - & 0.01 \\
\hline
\end{tabular}

\section{Discussion}

With the rising of quality and level of life of Chinese residents, and the increasing enhancement of people's health consciousness, the parents who emphasis on infant care is increasing, which makes great development in child health services. The development form is mainly embodied in the traditional health care model for change of modern health care model ${ }^{[3]}$. The infant period is the most important period for neonatal growth and development, at this stage giving the effective care measures has a great benefit on neonatal physical development and healthy growth, and has great influence on the future physical quality. Therefore, more and more parents attache great importance to the infant and child health, and the medical staff engaged in child care should also attach importance to children health, in order to promote the healthy growth of newborns, then to continuously enhance The physical quality of population in China ${ }^{[4]}$. Now, there are many clinical means and methods in child care, such as massage, massage, but Poor effect for separate implement. With the deepening of clinical research, it has been found that chiropractic massage technique can achieve a significant effect on children's health.Chiropractic massage is a traditional massage therapy in traditional Chinese medicine which belongs to the "external" scope in Chinese medicine theory. Early in two thousand years ago, the technique has been recorded in China famous medical experts, Alchemist Ge Hong's works "Prescriptions for Emergent Reference", in which it mentioned "pinch the spine skin", that is chiropractic which is recorded in future generations ${ }^{[5]}$. We know from the literature, the first chiropractic therapy is mainly used for the treatment of disease of the abdominal pain in adults, with the deepening of the scholars' studies in later ages, the therapy in the late Ming Dynasty begins disseminating civil, and for the treatment of infantile malnutrition related symptoms of therapy is referred to as "chiropractic", the therapy in the Ming and Qing Dynasties gradually 
mature and evolution for pediatric massage. With the large amount of research and practice of the later scholars, the indications of this therapy are increasing. Now the therapy is commonly used in treatment of children with respiratory tract infection, enuresis, malnutrition, moaning, indigestion, anorexia and other symptoms, and can also be used for children's health, to help its predecessor fitness, to promote their healthy growth ${ }^{[6]}$. Specific operation method of traditional chiropractic therapy: the ridge on the back is the central, which belongs to the Du meridian circulation moving parts, so often to Ridge back as part of the operation, along the ferrite Du meridian circulation route, by long strong point to Dazhui. Conventional operation method is "pinch three lift one", in the process, it needs the close cooperation of lifting, kneading, pushing the three methods, that is, in the process of pushing close combination of lifting and kneading technique ${ }^{[7]}$. In display of chiropractic, appropriate efforts will lift the skin, as the reference; and 3 times each knead will be put a hand rule "pinch three lift one; or only pinch is not provided, but in the end need to use double thumb kneading according to both sides of Shenshu Point $3 \sim 5 \mathrm{~min}$, the" kidney ". And the method also includes two methods, namely, "the two finger pinch method" and "three finger pinch method" ${ }^{[8]}$. Which finger pinch method, hands clenched into a fist, the bending deflection of the index finger side position with thumb abdominal anastomosis, in order to clamp the skin to display their chiropractic, side pinched edge goes to the pillow position. And "three finger pinch method of the middle finger, the forefinger and thumb finger ventral to, in order to clip to skin cast chiropractic, side pinched edge goes to pillow position $^{[9]}$. In the above mentioned techniques, which belongs to the "pinch three" manipulation stimulus quantity the strongest, while the three pinch is not handle lighter rules.

Chiropractic massage therapy mainly takes Chinese meridian, Qi and blood, yin and yang theory as the theoretical guidance, also makes syndrome differentiation and treatment method as the operating principle, in fact, the applied position Ridge back, relates to the bladder meridian of foot Taiyang and the governor vessel and so on. Records in the traditional Chinese medicine theory that spine is ferrite viscera Yin and Yang convergence, essence perfusion, meridian blood return, and chiropractic massage therapy by pinching the backs of ferrite, can stimulate ferrite Yang and promote systemic blood circulation, reconcile the Yin and Yang of ferrite gas, thus can achieve the purposes of illnesses and physical fitness ${ }^{[10-11]}$. And modern TCM clinical research found that the implement of chiropractic massage in child care can effectively stimulate the nerves and dry, in body neurohumoral effects, greatly enhance immune function of neonates; and also be bidirectional regulation of visceral activities, promote neonatal visceral well developed, especially more prominent management in the stomach and spleen effect. Also, the implement of chiropractic massage therapy in child care also greatly promote the growth and development and healthy growth of newborns, enhance the physical quality, prevent the ccurrence of various immunological disorders ${ }^{[14]}$.

In the study, the hospital implements standard massage on the nrwborns in group B. Carry on chiropractic massage on the newborns in group $\mathrm{A}$ on the basis of group $\mathrm{B}$, and results display that after the implementation, the clinical indicators of group A like weight, height and sleep time are better than that of group B before the implementation; before and after the implementation, the gain are greater than group $\mathrm{B}$; the total incidence of physical symptoms like anemia, malnutrition and rickets is lower than that in group B; the total incidence of adverse reaction like diarrhea, spits, pneumonia and red buttock was lower than that in group B, which is similar to the related research ${ }^{[15]}$. That tells us that on the basis of standard massage, chiropractic and massage technique can strengthen the health, increase the weight of newborns, prolong the sleep time, promote the growth and development, and reduce the occurrence of physical discomfort symptoms and adverse reactions of the newborns.

In summary, chiropractic massage therapy is the effective health care method in current child care, which can promote the growth and development of neonates, enhance the immunity, prevent various diseases, strengthen their physical quality. It has higher application value which is worthy to be popularized. 


\section{Acknowledgments}

Fund Project: innovation school of engineering, Guangzhou University City elected course the medical health care massage "teaching mode optimization research, NO.:A1-AFD015151Z1514.

\section{References}

[1] Yuan Zhan. The use of chiropractic massage techniques in health care for children, Modern Diagnosis and Treatment,2015,26(04):784-785.

[2] Hao Huiqiu. Curative effect observation of chiropractic massage for severe functional dyspepsia with anorexia ,Journal of Sichuan of Traditional Medicine, 2016, 30(01):194-196.

[3] Rong Bin. Comparison of clinical effect in the treatment of infantile anorexia between traditional massage therapy and syndrome differentiation and acupoint selection manipulation, Henan Traditional Chinese Medicine,2015,35(12):3168-3170.

[4] Zhu Xuan. Clinical study on the treatment of infantile anorexia with Chinese massage, Digest World Latest Med Inf, 2016, 16(08):202-205.

[5] Zhang Yan, Sun Anda, Zhao Shang. Clinical observation of 70 cases of infantile anorexia with stomach stagnation in different massage therapy, Modern Medicine and Health, 2016, 32(04): 589-600.

[6] Zhang Xiaolei, Sun Anda. Clinical observation of 65 cases of wind-heat in Children with massage treatment, Clinical Journal of Traditional Chinese Medicine, 2014, 12(06):9.

[7] Shen Yingpeng, Meng Qingzhu, Meng Lingzhai, ect. Effect of massage on recurrent respiratory tract infection in Children and its effect on serum immunoglobulin, Shandong Medical Journal,2015,55(17): 82-84.

[8] Shen Yingpeng, Meng Lingzhai, Liu Zhijun. The clinical research of the treatment of recurrent respiratory tract infection in Children with the combination of Chinese herbal medicine goldscreen powder with point massage, Journal of Liaoning University of Traditional Chinese Medicine,2014, 16(07):110-112.

[9] Dong Sheng, Guo Qinyuan, Fang Meilan, etc. Treatment of 80 cases of the treatment of recurrent respiratory tract infection in Children with massage and traditional Chinese medicine bath, Journal of External Therapy of Traditional Chinese Medicine,2013,22(01):22-23.

[10] Yan Shuanglan, Liu Xin. Massage therapy for 37 cases of recurrent respiratory tract infection in Children, Hubei Journal of Traditional Chinese Medicine,2014,36(02):60-61.

[11] Liu Li, Yang Wenting, Zheng Yulan. Clinical study on Weiling Decoction assisted massage in treating children with acute non bacterial diarrhea, Chinese Journal of Traditional Chinese Medicine,2014,20(07):1002-1003.

[12] Zong Jian, Zhang Hui. With glass injection combined Mao Ning Jing Lu Fen Tuina treatment of deficiency of rotavirus enteritis in children after the bed Khan Temple, Clinical Research in China,2014,6(08): 18-19.

[13] Ding Lifeng, Li Youjin, Zhang Yuanhao etc..Clinical observation on the effect of treating infantile allergic cough with the combination of calming liver and nourishing lung and relieving cough, Shanghai Journal of Traditional Chinese Medicine,2015,49(10):55-57.

[14] Quan Xuerong,Wang Jie,Shi Yajuan. Study on the rule of selecting points of massage therapy for recurrent respiratory tract infection (lung-spleen qi deficiency syndrome)in children, Journal of Tianjin university of Traditional Chinese Medicine,2013, 34(04):206-210.

[15] He Hui.Clinical observation of 45 cases of acupoint massage therapy in the treatment of enuresis in children, Chinese Community Doctors,2014,30(25):102. 